\title{
Solitary pulmonary capillary hemangioma presents as ground glass opacity on computed tomography indicating adenocarcinoma in situ/atypical adenomatous hyperplasia: A case report
}

\author{
YANMEI ZHU ${ }^{1}$, NING QU ${ }^{2}$, LILI SUN ${ }^{1}$, XIAO MENG ${ }^{1}$, XIAOYAN LI $^{1}$ and YONG ZHANG ${ }^{1}$ \\ Departments of ${ }^{1}$ Pathology and ${ }^{2}$ Radiology, Cancer Hospital of China Medical University, \\ Liaoning Cancer Hospital and Institute, Shenyang, Liaoning 110042, P.R. China
}

Received May 17, 2017; Accepted September 14, 2017

DOI: $10.3892 / b r .2017 .997$

\begin{abstract}
Solitary pulmonary capillary hemangioma $(\mathrm{SPCH})$ is a rare type of benign lung tumor, which must be distinguished from early lung cancer and precancerous lesions of the lung that manifest in a similar way upon imaging. The current study describes a case of SPCH and a review of the literature is performed. The patient was a 40-year-old Chinese woman who was referred to the Liaoning Cancer Hospital and Institute (Shenyang, China) in October, 2015 with a cough without obvious inducement. Computed tomography (CT) demonstrated pure ground glass opacity (GGO) in the right upper lung. Following systemic anti-inflammatory therapy over 6 months, the lesion did not exhibit any change on CT and was suspected to be an adenocarcinoma in situ (AIS) or atypical adenomatous hyperplasia (AAH). Video-assisted thoracic surgery wedge resection was subsequently performed. Frozen section diagnosis revealed a benign tumor without atypical epithelial cells. Subsequent to surgery, paraffin sections demonstrated that the tumor contained narrow alveolar cavities, thickened alveolar septa and a clear boundary separating it from healthy lung tissue. Furthermore, the proliferation lumens in the alveolar septa were lined with a single layer of flat cells. Immunohistochemical staining revealed that the flat cells were positive for cluster of differentiation CD31 and CD34, and negative for thyroid
\end{abstract}

Correspondence to: Professor Yong Zhang, Department of Pathology, Cancer Hospital of China Medical University, Liaoning Cancer Hospital and Institute, 44 Xiaoheyan Road, Shenyang, Liaoning 110042, P.R. China

E-mail: zhangyong@cancerhops-ln-cmu.com

Abbreviations: SPCH, solitary pulmonary capillary hemangioma; CT, computed tomography; GGO, ground glass opacity; AIS, adenocarcinoma in situ; AAH, atypical adenomatous hyperplasia; TTF1, thyroid transcription factor-1; CK, cytokeratin

Key words: solitary pulmonary capillary hemangioma, ground glass opacity, adenocarcinoma in situ, atypical adenomatous transcription factor-1 and cytokeratin. The proliferation of capillary vessels lead to the thickened alveolar septa and the tumor was diagnosed as SPCH. When imaging examination demonstrates a GGO in lung, SPCH must be considered in the differential diagnosis of AIS/AAH. As the prognosis of these lesions is entirely different, a pathological examination must be conducted to ensure a correct diagnosis.

\section{Introduction}

Solitary pulmonary capillary hemangioma $(\mathrm{SPCH})$ is a rare type of benign lung tumor that is difficult to diagnose due to a lack of symptoms, small lesion size and inconspicuous histological features (1). According to our search of previous literature, only $12 \mathrm{SPCH}$ cases have been reported (1-10). However, each of these studies recorded only 1 or 2 cases, and thus SPCH has not been studied in depth, which may explain the absence of a description of SPCH in the World Health Organization classification of lung tumors in 2015 (11).

$\mathrm{SPCH}$ is a benign lesion with an unclear etiology. In the previously reported cases, all patients were asymptomatic and the lesion was discovered incidentally rather than clinically (1-10). Additionally, tumor growth was universally slow and no patients succumbed from the disease (1-10). Improvements in understanding and recognition of SPCH by radiologists, surgeons and pathologists may aid to elucidate its epidemiology.

SPCH exhibits ground glass opacity (GGO) on computed tomography (CT), and the GGO lesion may be suspected to be an adenocarcinoma in situ (AIS)/atypical adenomatous hyperplasia (AAH) and/or to have focal inflammation (1). The prognosis and treatment of these lesions vary; SPCH is a benign lesion and may be treated by complete tumor resection, while AIS/AAH may be malignant or exhibit malignant potential and require chemotherapy and/or radiotherapy in addition to surgical resection. Meanwhile, inflammation is typically treated with anti-inflammatory therapy $(1,11)$. Thus, a detailed pathological examination is key to ensure correct diagnosis and subsequent treatment.

In the present study, a clinicopathological study of an $\mathrm{SPCH}$ case has been reported alongside a review of the literature. The purpose of the study was to define the clinical 
manifestation, radiological, histological and immunohistochemical features, and diagnosis of $\mathrm{SPCH}$, as a currently unrecognized lesion.

\section{Case report}

A 40-year-old Chinese woman was referred to the Liaoning Cancer Hospital and Institute (Shenyang, China) with a cough in October, 2015 without obvious inducement. The patient had no phlegm or dyspnea chest discomfort. The chest CT indicated a pure GGO lesion (size, 19x17 mm) in the right upper lung (Fig. 1). Laboratory findings, including tumor markers, such as carcinoembryonic antigen and carbohydrate antigen 19-9 were within the normal ranges at $1.7 \mathrm{ng} / \mathrm{ml}$ (normal range $0-5 \mathrm{ng} / \mathrm{ml}$ ) and $17.5 \mathrm{U} / \mathrm{ml}$ (normal range 0-37 U/ml), respectively (12). Following systemic anti-inflammatory therapy for 6 months, the size and density of the lesion had not changed according to CT. Although no abnormal uptake of fluorine-18-fluorodeoxyglucose in the lesion was observed during positron emission tomography-CT, it was suspected to be an AIS or AAH, and video-assisted thoracic surgery wedge resection was performed. Microscopically, a clear boundary between the tumor and normal lung tissue was observed in the low-power field (Fig. 2A). The tumor consisted of a narrow alveolar cavity, the alveolar septa was thickened with proliferated lumens that were various sizes and the lumens were lined with single-layer flat cells without atypical epithelial cells (Fig. 2B). Based on the above-mentioned findings, the intraoperative frozen sections demonstrated that the lesion was a benign tumor. Later, immunohistochemical staining of a paraffin section revealed the flat cells were positive for cluster of differentiation CD34 (Fig. 3A) and CD31 (Fig. 3B), and negative for D2-40, cytokeratin and thyroid transcription factor-1 (Fig. 3C). Therefore, the patient was diagnosed with $\mathrm{SPCH}$ and was followed up for 13 months after surgery with no recurrence.

\section{Discussion}

SPCH is a particularly rare type of benign tumor, characterized by the proliferation of capillary vessels. It was initially identified by Fugo et al (1) in 2006. Since then, only 13 cases have been reported (including the present case; Table I). There were seven males and six females, and the age ranged from 40 to 61 years (mean age, 52.8 years). The lesions were located on the left side in six cases and on the right side in seven cases. Two were located in the upper lobe, two in the middle lobe and nine cases were located in the lower lobe. The tumor size ranged from 7 to $20 \mathrm{~mm}$ (mean, $12 \mathrm{~mm}$ ). SPCH tends to present as a single small lesion, which is located in the lower lobe without any symptoms.

Observation of GGO is typical upon imaging of cases of SPCH (1). In 13 cases, four were pure GGO, and eight were mixed GGO and one case exhibited a solid nodule. The pure GGO may represent a limited proliferation of capillary vessels in the alveolar septum, which is followed by a thickened alveolar septum and a narrowed alveolar space, although the space was preserved and still contained air. The solid aspect of the GGO may be the result of the capillary vessel congestion,

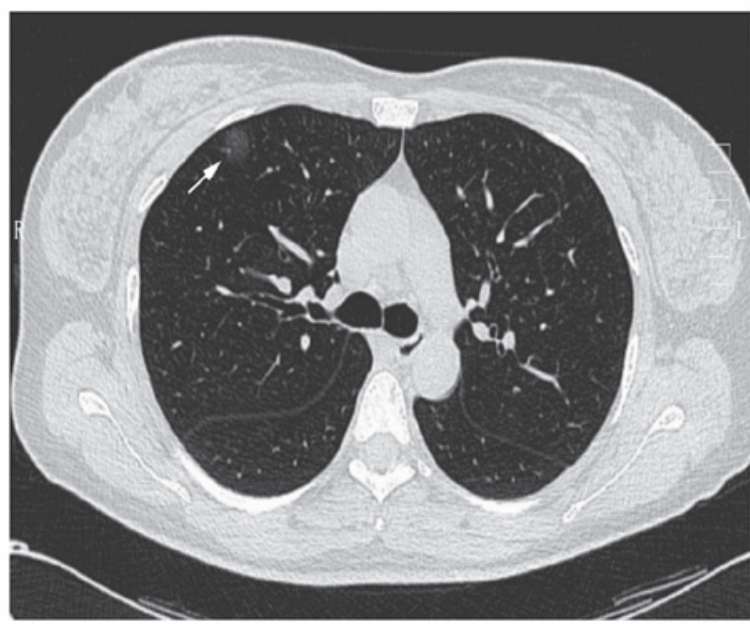

Figure 1. CT manifestation of the lung lesion. The chest CT exhibited a pure ground glass opacity lesion located in the subpleural area of the right upper lung, $\sim 19 \times 17 \mathrm{~mm}$ in size, with a clear boundary and no spiculations. CT, computed tomography.
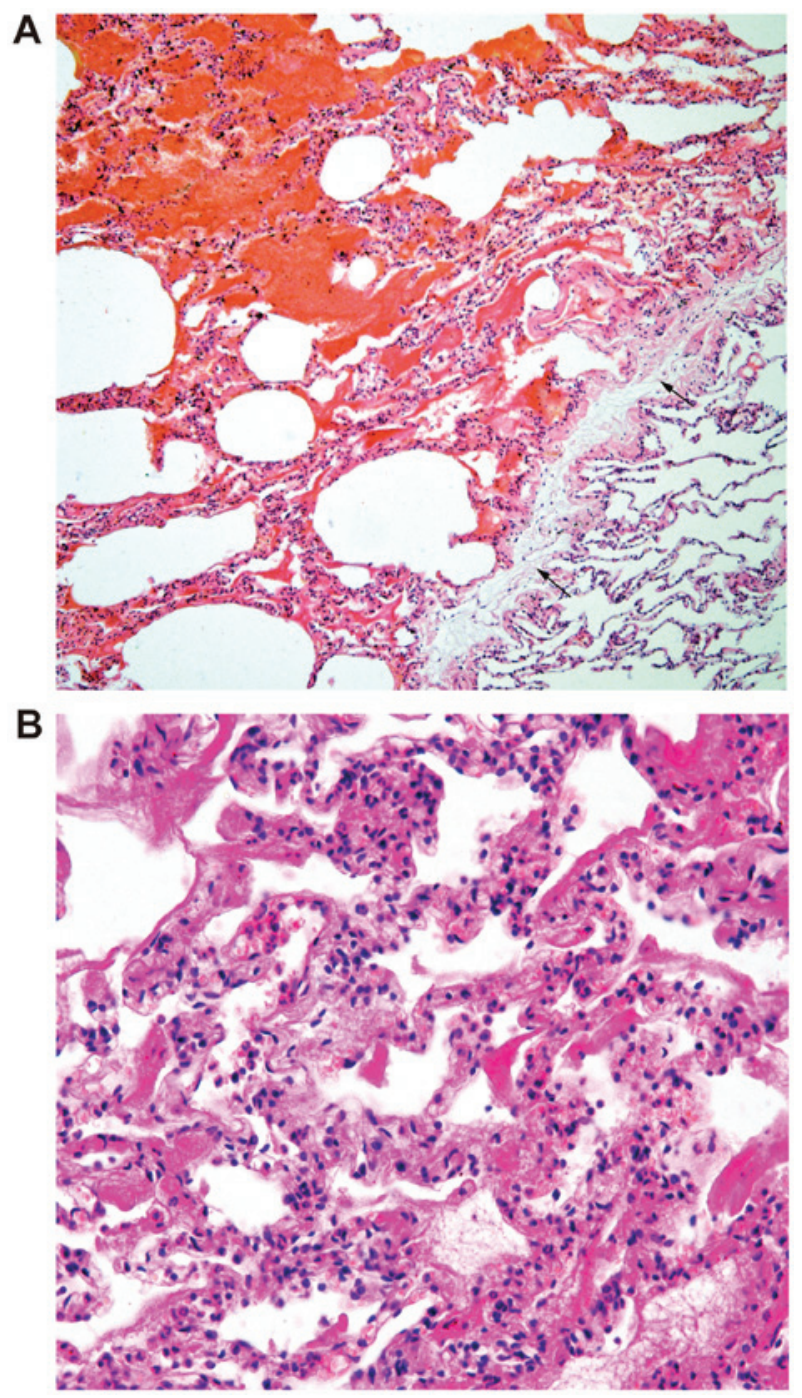

Figure 2. Morphological features of the lesion. (A) A clear boundary between the tumor and normal lung tissue is apparent (hematoxylin and eosin staining; magnification, $\mathrm{x} 40$ ). (B) The lesion exhibited thickening of the alveolar septa caused by the proliferation of lumens that were varied in size. The lumens were lined with single-layer flat cells without cytological atypia. The alveolar cavity had become narrowed (hematoxylin and eosin staining; magnification, x400). 


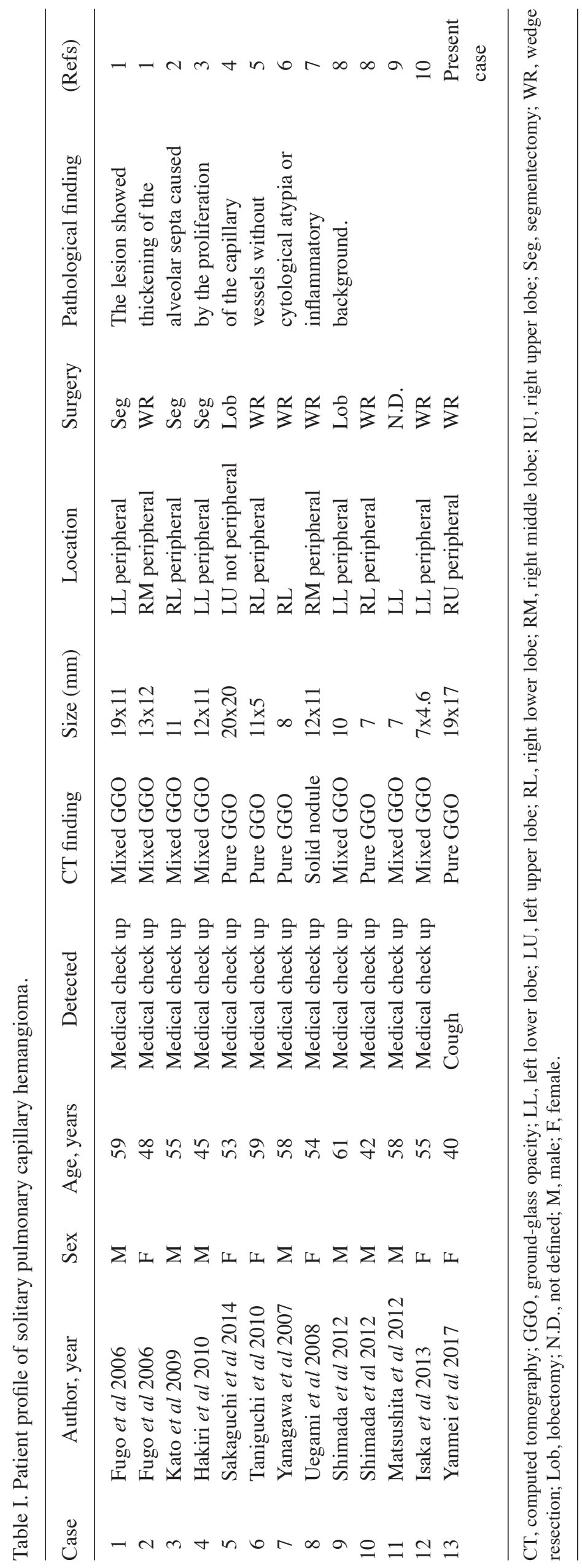


which may cause alveolar collapse and a resulting lack of space to contain air.

When GGO in the peripheral lung is observed, AIS/AAH may be highly suspected. It is difficult to distinguish AIS/AAH from SPCH by imaging examination alone. During surgery, Shimada et al (8) observed that the SPCH became impalpable following reiterative palpation. This finding may be caused by squeezing the blood in capillary vessels followed by constriction of the thickened alveolar septum and the lesion feeling the same as normal lung tissue. However, in AIS/AAH, based upon the different histopathological manifestations, the nodule would not be impalpable, as there are collagenous fibers or elastic fibers in the alveolar septa rather than capillary vessels. Therefore, when tumors are located in the peripheral lung, present with a GGO image and cannot be palpated following reiterative palpation during surgery, a diagnosis of SPCH may be determined. SPCH is a benign tumor, whereas AIS/AAH are malignant or have malignant potential. Therefore, it is critical to differentiate these lesions in the clinical setting.

Histopathology is an important method, as the histological appearance between SPCH and AIS/AAH is completely different (11). AIS often occurs in the peripheral lung, very close to the pleura. AIS is a small $(\leq 3 \mathrm{~cm})$ adenocarcinoma with growth restricted to neoplastic cells along pre-existing alveolar structure (lepidic growth), lacking stroma, vascular, alveolar space or pleural invasion. Grossly, it is a poorly defined nodule measuring up to $3 \mathrm{~cm}$ in size, with a tan or pale cut surface. Microscopically, AIS typically exhibits type II pneumocytes and/or Clara cell differentiation. The cells demonstrate marked cytologic atypia, such as dark and big nucleolus, coarse granular chromatin and pathologic mitosis. Alveolar septa widening is common in AIS, which is caused by sclerosis/elastosis rather than proliferation of capillary vessels (11). Macroscopically, $\mathrm{AAH}$ is a millimeter-sized, poorly defined, tan-yellowed nodule. It is characterized by mildly to moderately atypical type II pneumocyte and/or Clara cell proliferation along the alveolar walls. Double nuclei are common, but mitoses are extremely rare. There are gaps along the surface of the basement membrane between the cells. Alveolar septa widening is not obvious and there is no capillary vessel proliferation (11).

Pulmonary capillary hemangiomatosis $(\mathrm{PCH})$ is a multiple-lesion disease with numerous GGO nodules observed on CT (13). Although the histopathological characteristics are similar between PCH and SPCH, their clinical manifestations are completely different (14). PCH is a multiple-lesion disease with numerous GGO nodules on CT and is associated with pulmonary hypertension, whereas SPCH is a solitary nodular lesion with only solitary nodules observed on CT and the patient may present with mild or no symptoms (13). However, the underlying mechanisms have not been clarified (1). Evidence indicates that the increased expression levels of vascular endothelial growth factor and platelet-derived growth factor may be associated with PCH (1). However, the association between PCH and SPCH remains unknown. Based upon the similarities in histological manifestation, whether SPCH is the precursor lesion of $\mathrm{PCH}$ or whether they are independent of each other requires further investigation.

Pulmonary vein fibrosis is the main histological change observed in pulmonary veno-occlusive disease (PVOD) that leads to the complete blocking of pulmonary veins, venous stagnation

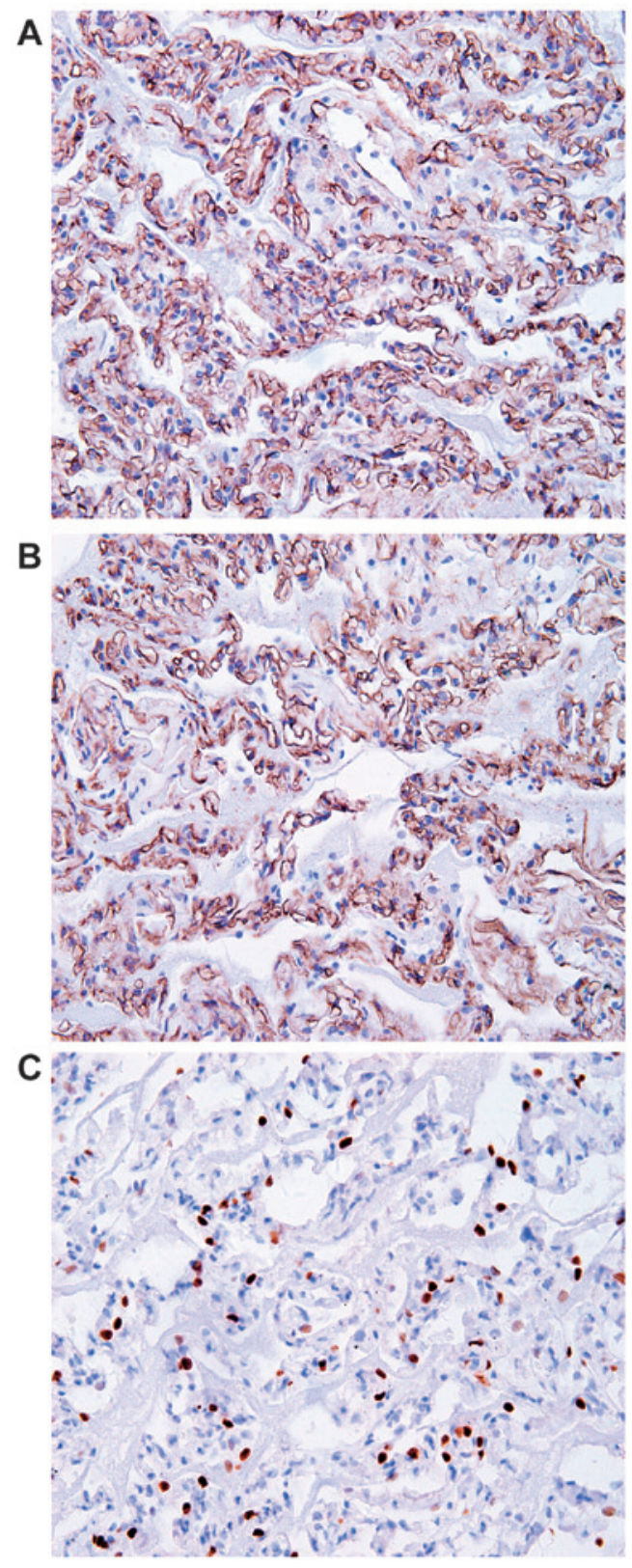

Figure 3. Immunohistochemical staining of the lesion. The flat cells were: (A) Positive for CD34 (DAB staining; magnification, x400), (B) positive for CD31 (DAB staining; magnification, $\mathrm{x} 400$ ) and $(\mathrm{C})$ negative for thyroid transcription factor-1 (DAB staining; magnification, $\mathrm{x} 400$ ). CD, cluster of differentiation; DAB, 3'-diaminobenzidine.

and alveolar wall capillary dilatation (14). The histological features of $\mathrm{PCH}$ are the proliferation of capillaries within the alveolar walls, as well as muscularization of arterioles and medial hypertrophy of muscular pulmonary arteries. Therefore, PCH causes pulmonary hypertension (14). PCH and PVOD are similar in clinical manifestation, though are fundamentally different diseases due to their histopathological features.

Focal inflammation also exhibits a GGO on CT (15). Clinically, it may decrease or disappear following antibiotic therapy. Histopathologically, inflammation results in different types of inflammatory cell, such as lymphocytes, plasmocytes, neutrophil granulocytes and proliferation of fibroblasts, rather than the proliferation of capillary vessels in widened alveolar septa (11). 
In conclusion, different types of lesion exhibit GGO on $\mathrm{CT}$, and the GGO lesion may be SPCH rather than AIS/AAH. The prognosis of these types of lesions is entirely different, therefore, a pathological examination must be conducted to ensure the correct diagnosis.

\section{Acknowledgements}

The current study was supported by the Dr. Scientific Research Start Fund Project in Liaoning Province, China (grant no. 20170520046).

\section{References}

1. Fugo K, Matsuno Y, Okamoto K, Kusumoto M, Maeshima A, Kaji M, Takabatake H, Kondo H and Moriyama N: Solitary capillary hemangioma of the lung: Report of 2 resected cases detected by high-resolution CT. Am J Surg Pathol 30: 750-753, 2006

2. Kato H, Oizumi H, Kanauchi N and Sadahiro M: A case of pulmonary capillary hemangioma diagnosed by thoraco-scopic segmentectomy. J Jpn Assoc Chest Surg 23: 932-935, 2009.

3. Hakiri S, Agatsuma $\mathrm{H}$ and Yoshioka $\mathrm{H}$ : A resected case of capillary hemangioma of the lung suspected to be lung cancer on chest computed tomography. Haigan 50: 841-845, 2010.

4. Sakaguchi Y, Isowa N, Tokuyasu $\mathrm{H}$ and Miura H: A resected case of solitary pulmonary capillary hemangioma showing pure ground glass opacity. Ann Thorac Cardiovasc Surg 20: 578-581, 2014.

5. Taniguchi D, Taniguchi H, Sano I, Tamura K, Shindou H, Shimizu K, Hamasaki K, Nakazaki T, Shigematsu K and Takahara O: Solitary capillary hemangioma in the lung: Report of a case. Kyobu Geka 63: 423-425, 2010 (In Chinese).

6. Yanagawa N, Kato H and Kanauchi N: Two cases of solitary peripheral small lung tumor needed to differentiate from small lung adenocarcinoma. Jpn J Diagn Pathol 24: 426-429, 2007.
7. Uegami S, Hirai S, Mitsui N, Matsuura Y and Hamanaka Y: A case of solitary capillary hemangioma of the lung. J Jpn Assoc Chest Surg 22: 641-644, 2008.

8. Shimada Y, Murakawa T, Sano A, Fukami T, Yoshida Y, Inoue Y, Morita S, Fukayama M and Nakajima J: Capillary hemangiomas of the lung presenting as ground glass opacities by high resolution computed tomography. Kyobu Geka 65: 1038-1043, 2012 (In Chinese).

9. Matsushita M, Kawakami S, Matsushita T, Sugiyama Y, Endo M, Shimojo H, Toishi M and Kadoya M: Changes in CT density of solitary capillary hemangioma of the lung upon varying patient position. Jpn J Radiol 30: 772-776, 2012.

10. Isaka T, Yokose T, Ito H, Washimi K, Imamura N, Watanabe M, Imai K, Nishii T, Yamada K, Nakayama H, et al: Case of solitary pulmonary capillary hemangioma: Pathological features based on frozen section analysis. Pathol Int 63: 615-618, 2013.

11. Travis WD, Brambilla E, Nicholson AG, Yatabe Y, Austin JHM, Beasley MB, Chirieac LR, Dacic S, Duhig E, Flieder DB, et al: WHO Panel: The 2015 World Health Organization Classification of Lung Tumors: Impact of Genetic, Clinical and Radiologic Advances Since the 2004 Classification. J Thorac Oncol 10: 1243-1260, 2015.

12. Chen XL and Chen XY: The significance of serum tumor markers in lung cancer diagnosis. J Clin Pulm Med 9: 590-592, 2004.

13. Li X, Jin ML, Wei P, Dai HP, Cui A, Zhang YG, Diao XL and Zhao HY: Pulmonary capillary hemangiomatosis: A clinicopathologic analysis of 2 cases with review of literature. Zhonghua Bing Li Xue Za Zhi 41: 16-19, 2012 (In Chinese).

14. Frazier AA, Franks TJ, Mohammed TL, Ozbudak IH and Galvin JR: From the archives of the AFIP: Pulmonary veno-occlusive disease and pulmonary capillary hemangiomatosis. Radiographics 27: 867-882, 2007.

15. Lv YG, Bao JH, Xu DU, Yan QH, Li YJ, Yuan DL and $\mathrm{Ma} \mathrm{JH}$ : Characteristic analysis of pulmonary ground-glass lesions with the help of 64-slice CT technology. Eur Rev Med Pharmacol Sci 21: 3212-3217, 2017. 